

\title{
A finiteness property of graded sequences of ideals
}

\author{
Mattias Jonsson and Mircea Mustață
}

Given a graded sequence of ideals $\left(\mathfrak{a}_{m}\right)_{m \geq 1}$ on $X$, having finite $\log$ canonical threshold, we show that if there are divisors $E_{m}$ over $X$ computing the log canonical threshold of $\mathfrak{a}_{m}$, and such that the log discrepancies of the divisors $E_{m}$ are bounded, then the set $\left\{E_{m} \mid m \geq 1\right\}$ is finite.

\section{Introduction}

Let $X$ be a smooth algebraic variety over an algebraically closed field $k$ of characteristic zero. The $\log$ canonical threshold of a nonzero ideal $\mathfrak{a}$ on $X$ is a fundamental invariant of the singularities of the subscheme defined by $\mathfrak{a}$. Originally known as the complex singularity index, it shows up in many contexts related to singularities, and it has found a plethora of applications in birational geometry [Kollár 1997; Ein and Mustaţă 2006].

In this note we will be interested in the behavior of this invariant in certain sequences of ideals. Let $\mathfrak{a}_{\mathbf{0}}=\left(\mathfrak{a}_{m}\right)_{m \geq 1}$ be a graded sequence of ideals on $X$, that is, a sequence of ideals that satisfies $\mathfrak{a}_{\ell} \cdot \mathfrak{a}_{m} \subseteq \mathfrak{a}_{\ell+m}$ for every $\ell, m \geq 1$. We always assume that, in addition, some ideal $\mathfrak{a}_{m}$ is nonzero. The main motivating example is the graded sequence $\mathfrak{a}^{L}$ associated to a line bundle $L$ of nonnegative Iitaka dimension on a smooth projective variety $X$ : the ideal $\mathfrak{a}_{m}^{L}$ defines the baselocus of the linear system $\left|L^{m}\right|$. Note that in this case the behavior of $\mathfrak{a}_{\bullet}^{L}$ is easy to understand if the section ring $\bigoplus_{m} \Gamma\left(X, L^{m}\right)$ is finitely generated over $k$. Indeed, in this case there is a positive integer $p$ such that $\mathfrak{a}_{m p}=\mathfrak{a}_{p}^{m}$ for all $m$. The study of $\mathfrak{a}_{\text {. }}^{L}$ is useful precisely when the section ring is not finitely generated (or at least, when this property is not known a priori).

Jonsson was partially supported by NSF grants DMS-0449465 and DMS-1001740. Mustață was partially supported by NSF grant DMS-0758454 and a Packard Fellowship.

MSC2010: primary 14F18; secondary 14B05.

Keywords: graded sequence of ideals, log canonical threshold. 


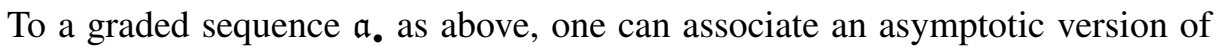
the log canonical threshold, by putting

$$
\operatorname{lct}\left(\mathfrak{a}_{\bullet}\right):=\sup _{m ; \mathfrak{a}_{m} \neq(0)} m \cdot \operatorname{lct}\left(\mathfrak{a}_{m}\right) .
$$

This can be infinite: for example, if $\mathfrak{a}_{\bullet}=\mathfrak{a}_{\bullet}^{L}$ as above, with $L$ big, then $\operatorname{lct}\left(\mathfrak{a}_{\bullet}\right)$ is infinite if and only if $L$ is nef (see Remark 2.2 below).

We will be concerned with the divisors that compute the log canonical thresholds of the elements of a graded sequence. We denote by $A\left(\operatorname{ord}_{E}\right)$ the log discrepancy of a divisor $E$ over $X$ (see Section 2 for the relevant definitions). Our main result, which gives a positive answer to a question of Mihai Păun, is Theorem A below. (Păun's question was motivated by [Siu 2009], in which Y.-T. Siu presents part of his arguments for the finite generation of the canonical ring. At the end of Section 6.3, he evokes a subtle point in his approach, involving the control of an infinite sequence of blow-ups. Although expressed in a different language, our main result shows that the infinite blow-up process in Siu's approach can be "stopped", provided that the log discrepancies of the divisors computing the log canonical thresholds are bounded.)

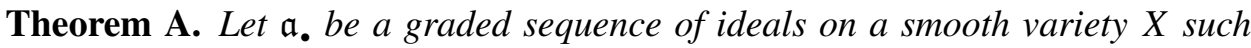
that $\operatorname{lct}\left(\mathfrak{a}_{\bullet}\right)<\infty$. If $I \subseteq \mathbb{Z}_{>0}$ is a subset such that for all $m \in I$ we have a divisor $E_{m}$ over $X$ that computes $\operatorname{lct}\left(\mathfrak{a}_{m}\right)$ such that $\left\{A\left(\operatorname{ord}_{E_{m}}\right) \mid m \in I\right\}$ is bounded, then the $\operatorname{set}\left\{E_{m} \mid m \in I\right\}$ is finite.

Corollary B. Under the hypothesis in Theorem A, suppose that the set I is infinite. Then there is a divisor $E$ over $X$ that computes $\operatorname{lct}\left(\mathfrak{a}_{m}\right)$ for infinitely $m$. In particular, E computes lct(a.).

In fact, since our proof will require replacing $X$ by a suitable blow-up, we will need to prove a stronger version of the above theorem, in which we replace the log canonical threshold by the possibly higher jumping numbers, in the sense of [Ein et al. 2004] (see Theorem 4.1 below for the precise statement).

Here is a sketch of the proof. Let $Z_{m}$ be the image of $E_{m}$ on $X$, and let $W$ be the Zariski closure of $\bigcup_{m \in I} Z_{m}$. We may assume that $W$ is irreducible, and we first show that since $\operatorname{lct}\left(\mathfrak{a}_{\bullet}\right)<\infty$, the asymptotic order of vanishing $\operatorname{ord}_{W}\left(\mathfrak{a}_{\bullet}\right)$ is positive. In particular, $W$ is a proper subset of $X$. If $W$ has codimension at least two in $X$, then blowing-up $X$ along $W$ decreases the log discrepancies of the divisors $E_{m}$, and since these are bounded above, we reduce to the case when $W$ is a hypersurface. In this case, we use the following result, which we believe is of independent interest.

Theorem C. Let $H$ be a hypersurface in $X$, and $\mathfrak{a}$ a nonzero ideal. Suppose that $E$ is a divisor over $X$ that computes $1 \mathrm{lt}(\mathfrak{a})$. If the image $Z$ of $E$ on $X$ is a proper 
subset of $H$, and if $H$ is smooth at the generic point of $Z$, then

$$
\operatorname{ord}_{Z}(\mathfrak{a}) \geq \operatorname{ord}_{H}(\mathfrak{a}) \cdot\left(1+\frac{\operatorname{ord}_{E}\left(I_{Z}\right)}{A\left(\operatorname{ord}_{E}\right)}\right)
$$

where $I_{Z}$ is the ideal defining $Z$.

Of course, as we have already mentioned, we need in fact a version of this result that applies also to higher jumping numbers (see Theorem 3.1 below for this more general version of the theorem). Using Theorem $C$, we show that if there were infinitely many $Z_{m}$ that were properly contained in $W$, then the ideals in $\mathfrak{a}$. would vanish along $W$ more than they should. Therefore all but finitely many of the $E_{m}$ are equal to $W$ (note that at this point we are on some blow-up of our original variety).

In the following section we review some basic facts about log canonical thresholds and higher jumping numbers. The proofs of the stronger versions of Theorems C and A are given in Section 3, and respectively, Section 4.

\section{Jumping numbers and valuations}

In this section we recall some definitions and results concerning the invariants of singularities that we will use, and set the notation for the rest of the paper. We work over a fixed algebraically closed field $k$ of characteristic zero. Let $X$ be a smooth variety over $k$ (in particular, we assume that $X$ is connected and separated). All ideal sheaves on $X$ are assumed to be coherent.

By a divisor $E$ over $X$ we mean a prime divisor on a normal variety $Y$ that has a proper birational morphism $\pi: Y \rightarrow X$. This induces a discrete valuation of the function field $K(Y)=K(X)$, that we denote by $\operatorname{ord}_{E}$. As usual, we identify two such divisors if they induce the same valuation. In particular, it follows from Hironaka's theorem on resolution of singularities that we may assume that both $Y$ and $E$ are nonsingular. If we denote by $K_{Y / X}$ the relative canonical divisor, then the log discrepancy of $\operatorname{ord}_{E}$ is given by $A\left(\operatorname{ord}_{E}\right):=1+\operatorname{ord}_{E}\left(K_{Y / X}\right)$. Note that this depends on the variety $X$, and whenever the variety is not clear from the context, we will write $A_{X}\left(\operatorname{ord}_{E}\right)$. The center of $E$ on $X$ is the image $c_{X}(E):=\pi(E)$ of $E$. We always consider on $c_{X}(E)$ the reduced scheme structure. If $\mathfrak{a}$ is a nonzero ideal sheaf on $X$, we put

$$
\operatorname{ord}_{E}(\mathfrak{a}):=\min \left\{\operatorname{ord}_{E}(f) \mid f \in \mathfrak{a} \cdot \mathscr{O}_{X, c_{X}(E)}\right\} \in \mathbb{R}_{\geq 0} .
$$

If $Z$ is the subscheme defined by $\mathfrak{a}$, we also denote this by $\operatorname{ord}_{E}(Z)$.

Given an irreducible closed subset $Z$ of $X$, we define the order of vanishing along $Z$ as follows. Consider the normalized blow-up of $X$ along $Z$, and put $\operatorname{ord}_{Z}:=\operatorname{ord}_{E}$, where $E$ is the unique irreducible component of the exceptional 
divisor that dominates $Z$. It is clear that in this case $c_{X}(E)=Z$. Note also that $\operatorname{ord}_{Z}(\mathfrak{a})=\min _{x \in Z} \operatorname{ord}_{x}(\mathfrak{a})$.

Let us recall the definition of multiplier ideals. For details and proofs we refer to [Lazarsfeld 2004, Section 9]. Suppose that $\mathfrak{a}$ is a nonzero ideal on $X$. Let $\mu: X^{\prime} \rightarrow X$ be a $\log$ resolution of $(X, \mathfrak{a})$, that is, $\pi$ is proper and birational, $X^{\prime}$ is nonsingular, $\mathfrak{a} \cdot \mathfrak{O}_{X^{\prime}}=\mathscr{O}_{X^{\prime}}(-F)$ for an effective divisor $F$, and $F+K_{X^{\prime} / X}$ has simple normal crossings. For every $\lambda \in \mathbb{R}_{\geq 0}$, the multiplier ideal of $\mathfrak{a}$ of exponent $\lambda$ is given by

$$
\mathscr{G}\left(\mathfrak{a}^{\lambda}\right):=\pi_{*} O_{X^{\prime}}\left(K_{X^{\prime} / X}-\lfloor\lambda F\rfloor\right) .
$$

The definition is independent of the choice of log resolution.

It is clear from the above definition that if $\lambda<\lambda^{\prime}$, then $\mathscr{g}\left(\mathfrak{a}^{\lambda^{\prime}}\right) \subseteq \mathscr{F}\left(\mathfrak{a}^{\lambda}\right)$. Furthermore, for every $\lambda$ there is $\varepsilon>0$ such that $\mathscr{g}\left(\mathfrak{a}^{\lambda}\right)=\mathscr{g}\left(\mathfrak{a}^{t}\right)$ for every $t \in[\lambda, \lambda+\varepsilon]$. One says that $\lambda>0$ is a jumping number of $\mathfrak{a}$ if $\mathscr{g}\left(\mathfrak{a}^{\lambda}\right) \neq \mathscr{g}\left(\mathfrak{a}^{\lambda^{\prime}}\right)$ for every $\lambda^{\prime}<\lambda$. It follows from the definition that if we write $F=\sum_{i} a_{i} E_{i}$, then for every jumping number $\lambda$ there is $i$ such that $\lambda a_{i}$ is an integer. In particular, the jumping numbers form a discrete set of rational numbers.

For basic properties of the jumping numbers and applications, we refer to [Ein et al. 2004]. The most important jumping number is the smallest one, known as the $\log$ canonical threshold and denoted by $\operatorname{lct}(\mathfrak{a})$. This is the smallest $\lambda$ such that $\mathscr{f}\left(\mathfrak{a}^{\lambda}\right) \neq \mathrm{O}_{X}$ (note that $\left.\mathscr{g}\left(\mathfrak{a}^{0}\right)=\mathrm{O}_{X}\right)$.

It is convenient to index the jumping numbers as follows (see [Jonsson and Mustaţă 2010]). Let $\mathfrak{q}$ be a nonzero ideal on $X$. We put

$$
\operatorname{lct}^{\mathfrak{q}}(\mathfrak{a}):=\min \left\{\lambda \mid \mathfrak{q} \not \subseteq \mathscr{(}\left(\mathfrak{a}^{\lambda}\right)\right\}
$$

Note that $\operatorname{lct}^{0_{X}}(\mathfrak{a})$ is the $\log$ canonical threshold $\operatorname{lct}(\mathfrak{a})$ of $\mathfrak{a}$. It follows from the definition that if $\mathfrak{a} \neq \mathscr{O}_{X}$, then $\bigcap_{\lambda \geq 0} \mathscr{F}\left(\mathfrak{a}^{\lambda}\right)=(0)$, hence $\operatorname{lct}^{\mathfrak{q}}(\mathfrak{a})$ is finite. When $\mathfrak{a}=\mathscr{O}_{X}$, we make the convention $\operatorname{lct}^{\mathfrak{q}}(\mathfrak{a})=\infty$. We will also use the notation $\operatorname{Arn}^{\mathfrak{q}}(\mathfrak{a}):=1 / \operatorname{lct}^{\mathfrak{q}}(\mathfrak{a})$ (where Arn stands for Arnold multiplicity). It follows from the definition that we have

$$
\operatorname{Arn}^{\mathfrak{q}}(\mathfrak{a})=\max _{E} \frac{\operatorname{ord}_{E}(\mathfrak{a})}{A\left(\operatorname{ord}_{E}\right)+\operatorname{ord}_{E}(\mathfrak{q})},
$$

where the maximum can be taken either over all divisors over $X$, or just over those lying on a $\log$ resolution of $(X, \mathfrak{a})$. We say that $E$ computes $\operatorname{lct}^{\mathfrak{q}}(\mathfrak{a})\left(\operatorname{or} \operatorname{Arn}^{\mathfrak{q}}(\mathfrak{a})\right)$ if the maximum in (1) is achieved by $E$.

The most interesting of the jumping numbers is the log canonical threshold. However, as the following lemma shows, the other jumping numbers appear naturally when we consider higher birational models. 
Proposition 2.1. Let $\pi: X^{\prime} \rightarrow X$ be a proper birational morphism, with $X^{\prime}$ smooth, and $\mathfrak{a}$ and $\mathfrak{q}$ nonzero ideals on $X$. If $\mathfrak{a}^{\prime}=\mathfrak{a} \cdot O_{X^{\prime}}$, and $\mathfrak{q}^{\prime}=\mathfrak{q} \cdot O_{X^{\prime}}\left(-K_{X^{\prime} / X}\right)$, then

$$
\operatorname{lct}^{\mathfrak{q}}(\mathfrak{a})=\operatorname{lct}^{\mathfrak{q}^{\prime}}\left(\mathfrak{a}^{\prime}\right)
$$

Proof. This is an immediate consequence of (1), and of the fact that for every divisor $E$ over $X$, we have $A_{X}\left(\operatorname{ord}_{E}\right)=A_{X^{\prime}}\left(\operatorname{ord}_{E}\right)+\operatorname{ord}_{E}\left(K_{X^{\prime} / X}\right)$.

Suppose now that $\mathfrak{a}_{\bullet}$ is a graded sequence of ideals on $X$, and let $S=\left\{m \mid \mathfrak{a}_{m} \neq\right.$ (0)\}. Note that $S$ is closed under addition. In this case we have the following asymptotic version of the jumping numbers:

$$
\operatorname{lct}^{\mathfrak{q}}\left(\mathfrak{a}_{\bullet}\right):=\sup _{m \in S} m \cdot \operatorname{lct}^{\mathfrak{q}}\left(\mathfrak{a}_{m}\right)=\lim _{m \rightarrow \infty, m \in S} m \cdot \operatorname{lct}^{\mathfrak{q}}\left(\mathfrak{a}_{m}\right)
$$

(see [Jonsson and Mustaţă 2010, Section 2]). We put $\operatorname{Arn}^{\mathfrak{q}}\left(\mathfrak{a}_{\bullet}\right)=1 / \operatorname{lct}^{\mathfrak{q}}\left(\mathfrak{a}_{\bullet}\right)$. When $\mathfrak{q}=\mathfrak{O}_{X}$, we simply write $\operatorname{lct}\left(\mathfrak{a}_{\text {. }}\right)$ and $\operatorname{Arn}\left(\mathfrak{a}_{\mathbf{0}}\right)$. Note that $\operatorname{lct}^{\mathfrak{q}}\left(\mathfrak{a}_{\mathbf{0}}\right) \in \mathbb{R}_{>0} \cup\{\infty\}$. One can show that $\operatorname{lct}^{\mathfrak{q}}\left(\mathfrak{a}_{\bullet}\right)=\infty$ if and only if $\operatorname{lct}\left(\mathfrak{a}_{\bullet}\right)=\infty$ (see [Jonsson and Mustaţă 2010, Corollary 6.10]).

Remark 2.2. If $X$ is a smooth projective variety, $L$ is a big line bundle on $X$, and $\mathfrak{a}_{\bullet}=\mathfrak{a}^{L}$ is the graded sequence of ideals defining the base loci of the powers of $L$ (see Introduction), then [Ein et al. 2006, Corollary 2.10] shows that $\operatorname{lct}\left(\mathfrak{a}_{\bullet}\right)=\infty$ if and only if $L$ is nef.

If $\mathfrak{a}_{\bullet}$ is as above and $E$ is a divisor over $X$, we will also consider the following asymptotic version of the order of vanishing along $E$ :

$$
\operatorname{ord}_{E}\left(\mathfrak{a}_{\bullet}\right):=\inf _{m} \frac{\operatorname{ord}_{E}\left(\mathfrak{a}_{m}\right)}{m}=\lim _{m \rightarrow \infty, m \in S} \frac{\operatorname{ord}_{E}\left(\mathfrak{a}_{m}\right)}{m} .
$$

We have the following extension of (1)

$$
\operatorname{Arn}^{\mathfrak{q}}\left(\mathfrak{a}_{\bullet}\right)=\sup _{E} \frac{\operatorname{ord}_{E}\left(\mathfrak{a}_{\bullet}\right)}{A\left(\operatorname{ord}_{E}\right)+\operatorname{ord}_{E}(\mathfrak{q})}
$$

For these facts, we refer to [Jonsson and Mustaţă 2010, Section 2]. We say that $E$ computes $\operatorname{lct}^{\mathfrak{q}}\left(\mathfrak{a}_{\bullet}\right)$ if the supremum in (3) is achieved by $E$. Note however that

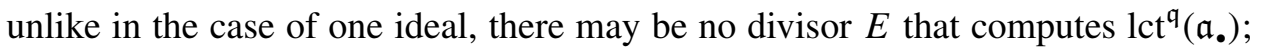
see [Jonsson and Mustaţă 2010, Example 8.5].

We will use the following Izumi-type estimate [Izumi 1985; Ein et al. 2003].

Proposition 2.3. If $E$ is a divisor over $X$ with $c_{X}(E)=Z$, then

$$
\operatorname{ord}_{E}(\mathfrak{a}) \leq A\left(\operatorname{ord}_{E}\right) \cdot \operatorname{ord}_{Z}(\mathfrak{a})
$$

for every nonzero ideal sheaf $\mathfrak{a}$ on $X$. 
Proof. We may replace $X$ by an affine open subset of the generic point of $Z$, and therefore assume that $X$ is affine. In this case we may assume that $\mathfrak{a}$ is principal. If $\operatorname{ord}_{Z}(\mathfrak{a})=m$, then for a general $p \in Z$ we have $\operatorname{ord}_{p}(\mathfrak{a})=m$. By [Kollár 1997, Lemma 8.10], there is an open neighborhood $U$ of $p$ such that $\operatorname{lct}\left(\left.\mathfrak{a}\right|_{U}\right) \geq 1 / m$, and we get the assertion in the proposition since $U \cap Z \neq \varnothing$ implies

$$
\frac{A\left(\operatorname{ord}_{E}\right)}{\operatorname{ord}_{E}(\mathfrak{a})} \geq \operatorname{lct}\left(\left.\mathfrak{a}\right|_{U}\right) \text {. }
$$

\section{An inequality between orders of vanishing}

We keep the notation and the conventions from Section 2. The following is the main result in this section. Note that in the special case $\mathfrak{q}=\mathfrak{O}_{X}$, this recovers Theorem $\mathrm{C}$ in the Introduction.

Theorem 3.1. Let $H$ be a hypersurface in $X$, and $\mathfrak{a}, \mathfrak{q}$ nonzero ideals on $X$. Suppose that $E$ is a divisor over $X$ that computes $\operatorname{lct}^{\mathfrak{q}}(\mathfrak{a})$. If the center $Z$ of $E$ on $X$ is a proper subset of $H$, and if $H$ is smooth at the generic point of $Z$, then the following inequality holds

$$
\operatorname{ord}_{Z}(\mathfrak{a}) \geq \operatorname{ord}_{H}(\mathfrak{a}) \cdot\left(1+\frac{\operatorname{ord}_{E}(Z)}{A\left(\operatorname{ord}_{E}\right)\left(1+\operatorname{ord}_{H}(\mathfrak{q})\right)}\right) .
$$

We start by recalling a basic estimate for the log discrepancy of a valuation. For a proof, see for example [Lazarsfeld 2004, page 157].

Lemma 3.2. Let $E$ be a divisor over $X$ with $c_{X}(E)=Z$, and let $\xi$ be the generic point of $Z$. If $x_{1}, \ldots, x_{r}$ form a regular system of parameters of $\mathfrak{O}_{X, \xi}$, then

$$
A\left(\operatorname{ord}_{E}\right) \geq \sum_{i=1}^{r} \operatorname{ord}_{E}\left(x_{i}\right) .
$$

Corollary 3.3. If $H$ is a hypersurface in $X$, and $E$ is a divisor over $X$ such that $Z:=c_{X}(E)$ is a proper subset of $H$, and $H$ is smooth at the generic point of $Z$, then

$$
A\left(\operatorname{ord}_{E}\right) \geq \operatorname{ord}_{E}(H)+\operatorname{ord}_{E}(Z) .
$$

Proof. Let $\xi$ be the generic point of $Z$. Since $H$ is smooth at $\xi$, we may choose a regular system of parameters $x_{1}, \ldots, x_{r}$ of $\mathrm{O}_{X, \xi}$ such that $H$ is defined at $\xi$ by $\left(x_{1}\right)$. Note that by assumption $r \geq 2$. By definition, we have $\operatorname{ord}_{E}(Z)=\min _{j} \operatorname{ord}_{E}\left(x_{j}\right)$. Let $i$ be such that $\operatorname{ord}_{E}\left(x_{i}\right)=\operatorname{ord}_{E}(Z)$. If $i \geq 2$, then by the lemma

$$
A\left(\operatorname{ord}_{E}\right) \geq \operatorname{ord}_{E}\left(x_{1}\right)+\operatorname{ord}_{E}\left(x_{i}\right)=\operatorname{ord}_{E}(H)+\operatorname{ord}_{E}(Z) .
$$

On the other hand, if $i=1$, then using again the lemma we get

$$
A\left(\operatorname{ord}_{E}\right) \geq \operatorname{ord}_{E}\left(x_{1}\right)+\operatorname{ord}_{E}\left(x_{2}\right) \geq 2 \cdot \operatorname{ord}_{E}\left(x_{1}\right)=\operatorname{ord}_{E}(H)+\operatorname{ord}_{E}(Z) .
$$


Proof of Theorem 3.1. Let us put $m=\operatorname{ord}_{H}(\mathfrak{a})$ and $p=\operatorname{ord}_{H}(\mathfrak{q})$. We can write $\mathfrak{a}=\mathrm{O}_{X}(-H)^{m} \cdot \tilde{\mathfrak{a}}$, and we get

$$
\operatorname{ord}_{E}(\mathfrak{a})=m \cdot \operatorname{ord}_{E}(H)+\operatorname{ord}_{E}(\tilde{\mathfrak{a}}), \quad \operatorname{ord}_{Z}(\mathfrak{a})=m+\operatorname{ord}_{Z}(\tilde{\mathfrak{a}})
$$

(note that $\operatorname{ord}_{Z}(H)=1$ since $H$ is smooth at the generic point of $Z$ ). Since $E$ computes $\operatorname{lct}^{\mathfrak{q}}(\mathfrak{a})$, it follows from (1) that

$$
\frac{\operatorname{ord}_{E}(\mathfrak{a})}{A\left(\operatorname{ord}_{E}\right)+\operatorname{ord}_{E}(\mathfrak{q})} \geq \frac{\operatorname{ord}_{H}(\mathfrak{a})}{A\left(\operatorname{ord}_{H}\right)+\operatorname{ord}_{H}(\mathfrak{q})}=\frac{m}{1+p} .
$$

Corollary $3.3 \operatorname{gives} \operatorname{ord}_{E}(H) \leq A\left(\operatorname{ord}_{E}\right)-\operatorname{ord}_{E}(Z)$, and combining this with (7) we deduce

$$
\begin{aligned}
m & \leq(1+p) \cdot \frac{\operatorname{ord}_{E}(\mathfrak{a})}{A\left(\operatorname{ord}_{E}\right)+\operatorname{ord}_{E}(\mathfrak{q})} \leq \frac{\operatorname{ord}_{E}(\tilde{\mathfrak{a}})+m\left(A\left(\operatorname{ord}_{E}\right)-\operatorname{ord}_{E}(Z)\right)+p \cdot \operatorname{ord}_{E}(\mathfrak{a})}{A\left(\operatorname{ord}_{E}\right)+\operatorname{ord}_{E}(\mathfrak{q})} \\
& =m+\frac{\operatorname{ord}_{E}(\tilde{\mathfrak{a}})+p \cdot \operatorname{ord}_{E}(\mathfrak{a})-m\left(\operatorname{ord}_{E}(\mathfrak{q})+\operatorname{ord}_{E}(Z)\right)}{A\left(\operatorname{ord}_{E}\right)+\operatorname{ord}_{E}(\mathfrak{q})}
\end{aligned}
$$

Therefore $\operatorname{ord}_{E}(\tilde{\mathfrak{a}}) \geq m\left(\operatorname{ord}_{E}(\mathfrak{q})+\operatorname{ord}_{E}(Z)\right)-p \cdot \operatorname{ord}_{E}(\mathfrak{a})$. Using one more time the first equation in (6), this implies

$$
(1+p) \cdot \operatorname{ord}_{E}(\tilde{\mathfrak{a}}) \geq m\left(\operatorname{ord}_{E}(\mathfrak{q})+\operatorname{ord}_{E}(Z)\right)-p m \cdot \operatorname{ord}_{E}(H) .
$$

On the other hand, by Proposition 2.3 we have $\operatorname{ord}_{E}(\tilde{\mathfrak{a}}) \leq A\left(\operatorname{ord}_{E}\right) \cdot \operatorname{ord}_{Z}(\tilde{\mathfrak{a}})$, while clearly $\operatorname{ord}_{E}(\mathfrak{q}) \geq p \cdot \operatorname{ord}_{E}(H)$. Putting these together with (9) gives

$$
\begin{aligned}
(1+p) A\left(\operatorname{ord}_{E}\right) \cdot \operatorname{ord}_{Z}(\tilde{\mathfrak{a}}) & \geq(1+p) \cdot \operatorname{ord}_{E}(\tilde{\mathfrak{a}}) \\
& \geq m\left(\operatorname{ord}_{E}(\mathfrak{q})+\operatorname{ord}_{E}(Z)\right)-p m \cdot \operatorname{ord}_{E}(H) \\
& \geq m \cdot \operatorname{ord}_{E}(Z) .
\end{aligned}
$$

Combining this with the second equality in (6), we obtain

$$
\operatorname{ord}_{Z}(\mathfrak{a})=m+\operatorname{ord}_{Z}(\tilde{\mathfrak{a}}) \geq m \cdot\left(1+\frac{\operatorname{ord}_{E}(Z)}{A\left(\operatorname{ord}_{E}\right)(1+p)}\right)
$$

which completes the proof of the theorem.

Remark 3.4. In Theorem 3.1 one can replace $\operatorname{ord}_{E}$ by any real valuation of $K(X)$, having center on $X$ and computing $\operatorname{lct}^{\mathfrak{q}}(\mathfrak{a})$. The proof goes through if one uses the definition of $A(v)$ from [Jonsson and Mustață 2010, Section 5]. In this case, the assertion in Lemma 3.2 follows from Corollary 5.4 of that reference.

Example 3.5. The inequality in Theorem 3.1 is optimal, at least in an asymptotic sense. Indeed, let us consider the ideal $\mathfrak{a}=x^{m}\left(x, y^{m+1}\right)$ in $k[x, y]$, where $m$ is a positive integer. Since this is a monomial ideal, one can use Howald's theorem [Howald 2001] to compute its log canonical threshold. It is easy to check that 
$\operatorname{lct}(\mathfrak{a})=(m+2) /(m+1)^{2}$, and this $\log$ canonical threshold is computed by the (toric) divisor $E$ over $X=\mathbb{A}^{2}$ such that

$$
\operatorname{ord}_{E}\left(\sum_{i, j \geq 0} c_{i, j} x^{i} y^{j}\right)=\min \left\{(m+1) i+j \mid c_{i, j} \neq 0\right\} .
$$

Note that $A\left(\operatorname{ord}_{E}\right)=m+2$, and the center of $E$ on $X$ is the origin. If we take $\mathfrak{q}=\mathrm{O}_{X}$ and $H=(x=0)$, then

$$
\frac{\operatorname{ord}_{Z}(\mathfrak{a})}{\operatorname{ord}_{H}(\mathfrak{a})\left(1+\frac{\operatorname{ord}_{E}(Z)}{A\left(\operatorname{ord}_{E}\right)}\right)}=\frac{m+1}{m\left(1+\frac{1}{m+2}\right)}=\frac{(m+1)(m+2)}{m(m+3)},
$$

and this converges to 1 when $m$ goes to infinity.

Remark 3.6. The right-hand side of the inequality (4) is bounded above by

$$
\operatorname{ord}_{H}(\mathfrak{a}) \cdot\left(1+\frac{1}{\operatorname{lct}\left(I_{Z}\right) \cdot\left(1+\operatorname{ord}_{H}(\mathfrak{q})\right)}\right)
$$

where $I_{Z}$ is the ideal defining $Z$. One could ask whether this expression is $\leq$ $\operatorname{ord}_{Z}(\mathfrak{a})$, improving in this way the assertion in Theorem 3.1. However, this is not the case: let us consider the special case $m=3$ in Example 3.5, that is, $\mathfrak{a}=$ $x^{3}\left(x, y^{4}\right)$. With $\mathfrak{q}=O_{X}$ and $H=(x=0)$, we have $\operatorname{ord}_{Z}(\mathfrak{a})=4$, while

$$
\operatorname{ord}_{H}(\mathfrak{a}) \cdot\left(1+\frac{1}{\operatorname{lct}\left(I_{Z}\right)}\right)=3\left(1+\frac{1}{2}\right)=\frac{9}{2}>4 .
$$

\section{The main result}

In this section we prove the generalized version of Theorem A in the Introduction. We work in the same setting as in Section 2.

Theorem 4.1. Let $\mathfrak{a}$. be a graded sequence of ideals on $X$, and $\mathfrak{q}$ a nonzero ideal on $X$ such that $\operatorname{lct}^{\mathfrak{q}}\left(\mathfrak{a}_{\bullet}\right)<\infty$. If $I \subseteq \mathbb{Z}_{>0}$ is a subset such that for all $m \in I$ we have a divisor $E_{m}$ over $X$ that computes $\operatorname{lct}^{\mathfrak{q}}\left(\mathfrak{a}_{m}\right)$ such that $\left\{A\left(\operatorname{ord}_{E_{m}}\right) \mid m \in I\right\}$ is bounded, then the set $\left\{E_{m} \mid m \in I\right\}$ is finite.

Corollary 4.2. Under the same hypothesis as in Theorem 4.1, suppose that the set $I$ is infinite. Then there is a divisor $E$ over $X$ that computes $\operatorname{lct}^{\mathfrak{q}}\left(\mathfrak{a}_{m}\right)$ for infinitely many $m$. In particular, E computes $\operatorname{lct}^{\mathfrak{q}}\left(\mathfrak{a}_{\bullet}\right)$.

Proof of Theorem 4.1. Note that the hypothesis implies, in particular, that $\mathfrak{a}_{m}$ is nonzero for every $m \in I$. We assume that $I$ is an infinite set, that $E_{i} \neq E_{j}$ for all $i \neq j$ in $I$ and aim to derive a contradiction. Let $Z_{m}=c_{X}\left(E_{m}\right)$. We argue by induction on $M:=\max \left\{A\left(\operatorname{ord}_{E_{i}}\right) \mid i \in I\right\}$. This is finite by assumption. Note that $M$ is a positive integer, and $M=1$ if and only if all the $E_{i}$ 's are divisors on $X$. At 
several stages in the proof we will replace $I$ by an infinite subset. Note that this can only decrease the value of $M$.

We start with the following lemma.

Lemma 4.3. With the above notation, suppose that there is an infinite subset $J \subseteq I$ such that $W:=\overline{\bigcup_{j \in J} Z_{j}}$ is irreducible, and $Z_{j} \neq W$ for all $j \in J$. In this case

$$
\operatorname{ord}_{W}\left(\mathfrak{a}_{\bullet}\right) \geq \operatorname{Arn}\left(\mathfrak{a}_{\bullet}\right) \geq \operatorname{Arn}^{\mathfrak{q}}\left(\mathfrak{a}_{\bullet}\right)>0 .
$$

Proof. We only need to prove the first inequality. Let $C=\operatorname{Arn}\left(\mathfrak{a}_{\bullet}\right)$, so that $\operatorname{Arn}\left(\mathfrak{a}_{m}\right) \geq C m$ for every $m$. If $j \in J$, then by Proposition 2.3 we have $\operatorname{Arn}\left(\mathfrak{a}_{j}\right) \leq$ $\operatorname{ord}_{Z_{j}}\left(\mathfrak{a}_{j}\right)$.

We need to show that $\operatorname{ord}_{W}\left(\mathfrak{a}_{m}\right) \geq C m$ for every $m \geq 1$. We may, of course, assume that $\mathfrak{a}_{m}$ is nonzero. By hypothesis, we can find $0 \leq \ell \leq m-1$ such that the set

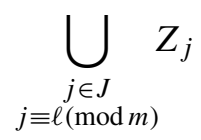

is dense in $W$. Since all $Z_{j}$ are proper subsets of $W$, this implies that if in (10) we only take the union over those $j \in J$ with $j \equiv \ell(\bmod m)$ and with $j \geq N$, for some $N$, then the union is still dense in $W$. Let us fix $j_{0} \in J$ with $j_{0} \equiv \ell(\bmod m)$, and let $C^{\prime}:=\max _{x \in W} \operatorname{ord}_{x}\left(\mathfrak{a}_{j_{0}}\right)<\infty$ (recall that $\mathfrak{a}_{j_{0}}$ is nonzero). If $m p+j_{0} \in J$, then the inclusion $\mathfrak{a}_{m}^{p} \cdot \mathfrak{a}_{j_{0}} \subseteq \mathfrak{a}_{m p+j_{0}}$ implies

$p \cdot \operatorname{ord}_{Z_{m p+j_{0}}}\left(\mathfrak{a}_{m}\right)+\operatorname{ord}_{Z_{m p+j_{0}}}\left(\mathfrak{a}_{j_{0}}\right) \geq \operatorname{ord}_{Z_{m p+j_{0}}}\left(\mathfrak{a}_{m p+j_{0}}\right) \geq \operatorname{Arn}\left(\mathfrak{a}_{m p+j_{0}}\right) \geq C\left(m p+j_{0}\right)$.

Therefore $\operatorname{ord}_{Z_{m p+j_{0}}}\left(\mathfrak{a}_{m}\right) \geq C m-C^{\prime} / p$. Since we have arbitrarily large such $p$, and since the union of the corresponding $Z_{m p+j_{0}}$ is dense in $W$, we conclude that $\operatorname{ord}_{W}\left(\mathfrak{a}_{m}\right) \geq C m$, as required.

A first consequence of the lemma is that if $W$ is the closure of $\bigcup_{i \in I} Z_{i}$, then $W \neq X$. In particular, this shows that when $M=1$, we have a contradiction.

Arguing by Noetherian induction on $W$, we may assume that $W$ is minimal in $X$ with the property that there is an infinite family of divisors $\left(E_{i}\right)_{i \in I}$ as above, with $\max \left\{A\left(\operatorname{ord}_{E_{i}}\right) \mid i \in I\right\} \leq M$. This implies first that $W$ is irreducible. Indeed, if we consider the irreducible decomposition $W=W_{1} \cup \cdots \cup W_{r}$, then there is $j$ such that $Z_{i} \subseteq W_{j}$ for infinitely many $i \in I$. Since we may replace $I$ by $\left\{i \in I \mid Z_{i} \subseteq W_{j}\right\}$, it follows from the minimality assumption on $W$ that $W=W_{j}$.

A second consequence of the minimality of $W$ is that for every infinite subset $J \subseteq I$, the union $\bigcup_{j \in J} Z_{j}$ is dense in $W$. In particular, if $U$ is an open subset of $X$ that meets $W$, then there are infinitely many $i \in I$ such that $U$ meets $Z_{i}$ (and the union of these $Z_{i} \cap U$ is dense in $W \cap U$ ). Therefore in order to deduce a 
contradiction we may replace $X$ by $U$ and each $\mathfrak{a}_{m}$ by its restriction to $U$. We may thus assume that $W$ is nonsingular.

We claim that the induction hypothesis on $M$ implies that $W$ is a hypersurface in $X$. Indeed, suppose that $c=\operatorname{codim}(W, X) \geq 2$, and let $\pi: X^{\prime} \rightarrow X$ be the blow-up of $X$ along $W$. If $E$ is the exceptional divisor of $\pi$, then $K_{X^{\prime} / X}=(c-1) E$. Since $c_{X}\left(E_{i}\right) \subseteq W$ for every $i \in I$, it follows that $c_{X^{\prime}}\left(E_{i}\right) \subseteq E$, hence

$$
A_{X^{\prime}}\left(\operatorname{ord}_{E_{i}}\right)=A_{X}\left(\operatorname{ord}_{E_{i}}\right)-\operatorname{ord}_{E_{i}}\left(K_{X^{\prime} / X}\right) \leq A_{X}\left(\operatorname{ord}_{E_{i}}\right)-(c-1) .
$$

If $\mathfrak{a}_{m}^{\prime}=\mathfrak{a}_{m} \cdot \mathscr{O}_{X^{\prime}}$ and $\mathfrak{q}^{\prime}=\mathfrak{q} \cdot \mathscr{O}_{X^{\prime}}\left(-K_{X^{\prime} / X}\right)$, then by Proposition 2.1 we have $\operatorname{lct}^{\mathfrak{q}}\left(\mathfrak{a}_{i}\right)=$ $\operatorname{lct}^{\mathfrak{q}^{\prime}}\left(\mathfrak{a}_{i}\right)$, and it follows from hypothesis and (1) that $E_{i}$ computes $\operatorname{lct}^{\mathfrak{q}^{\prime}}\left(\mathfrak{a}_{i}^{\prime}\right)$ for every $i \in I$. Since $\max \left\{A_{X^{\prime}}\left(\operatorname{ord}_{E_{i}}\right) \mid i \in I\right\} \leq M-1$, we have a contradiction by induction on $M$.

Therefore $W$ is a smooth hypersurface in $X$. If $Z_{i}=W$, then $E_{i}=W$, hence this can be the case for at most one $i$. After discarding this $i$, we may assume that each $Z_{i}$ is a proper subset of $W$. In particular, we may apply Theorem 3.1 to get

$$
\operatorname{ord}_{Z_{i}}\left(\mathfrak{a}_{i}\right) \geq \operatorname{ord}_{W}\left(\mathfrak{a}_{i}\right) \cdot\left(1+\frac{\operatorname{ord}_{E_{i}}\left(Z_{i}\right)}{A\left(\operatorname{ord}_{E_{i}}\right)\left(1+\operatorname{ord}_{W}(\mathfrak{q})\right)}\right) .
$$

Note that $\operatorname{ord}_{E_{i}}\left(Z_{i}\right) \geq 1$ for all $i \in I$. Let $\alpha=\operatorname{ord}_{W}\left(\mathfrak{a}_{\bullet}\right)$. We have $\alpha>0$ by Lemma 4.3. Let us fix $\varepsilon>0$ with

$$
\varepsilon<\frac{1}{M\left(1+\operatorname{ord}_{W}(\mathfrak{q})\right)} .
$$

If we show that $\operatorname{ord}_{W}\left(\mathfrak{a}_{m}\right) \geq \alpha m(1+\varepsilon)$ for every $m \geq 1$, then

$$
\alpha=\operatorname{ord}_{W}\left(\mathfrak{a}_{\bullet}\right) \geq \alpha(1+\varepsilon),
$$

a contradiction. We now argue as in the proof of Lemma 4.3. Let $0 \leq \ell \leq m-1$ be such that the set in $(10)$ is dense in $W$. We fix $j_{0} \in I$ such that $j_{0} \equiv \ell(\bmod m)$, and let $C^{\prime}:=\max _{x \in W} \operatorname{ord}_{x}\left(\mathfrak{a}_{j_{0}}\right)$. It follows from the inclusion $\mathfrak{a}_{m}^{p} \cdot \mathfrak{a}_{j_{0}} \subseteq \mathfrak{a}_{m p+j_{0}}$ and from (11) that for every $p$ such that $m p+j_{0} \in I$ we have

$p \cdot \operatorname{ord}_{Z_{m p+j_{0}}}\left(\mathfrak{a}_{m}\right) \geq \operatorname{ord}_{Z_{m p+j_{0}}}\left(\mathfrak{a}_{m p+j_{0}}\right)-\operatorname{ord}_{Z_{m p+j_{0}}}\left(\mathfrak{a}_{j_{0}}\right) \geq \operatorname{ord}_{W}\left(\mathfrak{a}_{m p+j_{0}}\right)(1+\varepsilon)-C^{\prime}$.

Therefore for every such $p$ we have ord $Z_{Z_{m+j_{0}}}\left(\mathfrak{a}_{m}\right) \geq \alpha m(1+\varepsilon)-C^{\prime} / p$. Since there are arbitrarily large such $p$, and the union of the corresponding $Z_{m p+j_{0}}$ is dense in $W$, we conclude that $\operatorname{ord}_{W}\left(\mathfrak{a}_{m}\right) \geq \alpha m(1+\varepsilon)$. As we have seen, this leads to a contradiction, and thus completes the proof of the theorem.

\section{Acknowledgement}

We are grateful to Mihai Păun for the question that led to our main result. 


\section{References}

[Ein and Mustață 2006] L. Ein and M. Mustață, "Invariants of singularities of pairs”, pp. 583-602 in International congress of mathematicians, vol. 2, edited by M. Sanz-Solé et al., Eur. Math. Soc., Zürich, 2006. MR 2007m:14050 Zbl 1096.14030

[Ein et al. 2003] L. Ein, R. Lazarsfeld, and K. E. Smith, "Uniform approximation of Abhyankar valuation ideals in smooth function fields", Amer. J. Math. 125:2 (2003), 409-440. MR 2003m:13004 Zbl 1033.14030

[Ein et al. 2004] L. Ein, R. Lazarsfeld, K. E. Smith, and D. Varolin, "Jumping coefficients of multiplier ideals”, Duke Math. J. 123:3 (2004), 469-506. MR 2005k:14004 Zbl 1061.14003

[Ein et al. 2006] L. Ein, R. Lazarsfeld, M. Mustață, M. Nakamaye, and M. Popa, "Asymptotic invariants of base loci”, Ann. Inst. Fourier (Grenoble) 56:6 (2006), 1701-1734. MR 2007m:14008 Zbl 1127.14010

[Howald 2001] J. A. Howald, "Multiplier ideals of monomial ideals", Trans. Amer. Math. Soc. 353:7 (2001), 2665-2671. MR 2002b:14061 Zbl 0979.13026

[Izumi 1985] S. Izumi, "A measure of integrity for local analytic algebras", Publ. Res. Inst. Math. Sci. 21:4 (1985), 719-735. MR 87i:32014 Zbl 0587.32016

[Jonsson and Mustață 2010] M. Jonsson and M. Mustață, "Valuations and asymptotic invariants for sequences of ideals", preprint, 2010. To appear in Ann. Inst. Fourier. arXiv 1011.3699

[Kollár 1997] J. Kollár, "Singularities of pairs", pp. 221-287 in Algebraic geometry (Santa Cruz, 1995), vol. 1, edited by J. Kollár et al., Proc. Sympos. Pure Math. 62, Amer. Math. Soc., Providence, RI, 1997. MR 99m:14033 Zbl 0905.14002

[Lazarsfeld 2004] R. Lazarsfeld, Positivity in algebraic geometry, II: positivity for vector bundles, and multiplier ideals, Ergeb. Math. Grenzgeb. (3) 49, Springer, Berlin, 2004. MR 2005k:14001b Zbl 1093.14500

[Siu 2009] Y.-T. Siu, "Techniques for the analytic proof of the finite generation of the canonical ring”, pp. 177-219 in Current developments in mathematics, 2007, edited by D. Jerison et al., Int. Press, Somerville, MA, 2009. MR 2011c:32030 Zbl 1183.14012

Communicated by János Kollár

Received 2010-11-27 Revised 2011-05-23 Accepted 2011-06-30

mattiasj@umich.edu

mmustata@umich.edu

Department of Mathematics, University of Michigan, Ann Arbor, MI 48109-1043, United States

Department of Mathematics, University of Michigan, Ann Arbor, MI 48109-1043, United States 


\section{Algebra \& Number Theory}

msp.berkeley.edu/ant

\section{EDITORS}

MANAGING EDITOR

Bjorn Poonen

Massachusetts Institute of Technology

Cambridge, USA

\author{
EDITORIAL BOARD CHAIR \\ David Eisenbud \\ University of California \\ Berkeley, USA
}

\section{BOARD OF EDITORS}

Georgia Benkart

Dave Benson

Richard E. Borcherds

John H. Coates

J-L. Colliot-Thélène

Brian D. Conrad

Hélène Esnault

Hubert Flenner

Edward Frenkel

Andrew Granville

Joseph Gubeladze

Ehud Hrushovski

Craig Huneke

Mikhail Kapranov

Yujiro Kawamata

János Kollár

Yuri Manin

Barry Mazur

Philippe Michel

Susan Montgomery
University of Wisconsin, Madison, USA

University of Aberdeen, Scotland

University of California, Berkeley, USA

University of Cambridge, UK

CNRS, Université Paris-Sud, France

University of Michigan, USA

Universität Duisburg-Essen, Germany

Ruhr-Universität, Germany

University of California, Berkeley, USA

Université de Montréal, Canada

San Francisco State University, USA

Hebrew University, Israel

University of Kansas, USA

Yale University, USA

University of Tokyo, Japan

Princeton University, USA

Northwestern University, USA

Harvard University, USA

École Polytechnique Fédérale de Lausanne

University of Southern California, USA
Shigefumi Mori

Raman Parimala

Jonathan Pila

Victor Reiner

Karl Rubin

Peter Sarnak

Joseph H. Silverman

Michael Singer

Ronald Solomon

Vasudevan Srinivas

J. Toby Stafford

Bernd Sturmfels

Richard Taylor

Ravi Vakil

Michel van den Bergh

Marie-France Vignéras

Kei-Ichi Watanabe

Andrei Zelevinsky

Efim Zelmanov
RIMS, Kyoto University, Japan

Emory University, USA

University of Oxford, UK

University of Minnesota, USA

University of California, Irvine, USA

Princeton University, USA

Brown University, USA

North Carolina State University, USA

Ohio State University, USA

Tata Inst. of Fund. Research, India

University of Michigan, USA

University of California, Berkeley, USA

Harvard University, USA

Stanford University, USA

Hasselt University, Belgium

Université Paris VII, France

Nihon University, Japan

Northeastern University, USA

University of California, San Diego, USA

\section{PRODUCTION}

contact@msp.org

Silvio Levy, Scientific Editor

See inside back cover or www.jant.org for submission instructions.

The subscription price for 2012 is US \$175/year for the electronic version, and \$275/year (+\$40 shipping outside the US) for print and electronic. Subscriptions, requests for back issues from the last three years and changes of subscribers address should be sent to Mathematical Sciences Publishers, Department of Mathematics, University of California, Berkeley, CA 94720-3840, USA.

Algebra \& Number Theory (ISSN 1937-0652) at Mathematical Sciences Publishers, Department of Mathematics, University of California, Berkeley, CA 94720-3840 is published continuously online. Periodical rate postage paid at Berkeley, CA 94704, and additional mailing offices.

ANT peer review and production are managed by EditFLOW ${ }^{\circledR}$ from Mathematical Sciences Publishers.

PUBLISHED BY

mathematical sciences publishers

http://msp.org/

A NON-PROFIT CORPORATION

Typeset in IAT $_{\mathrm{E}} \mathrm{X}$

Copyright (C2012 by Mathematical Sciences Publishers 


\section{Algebra \& Number Theory}

Volume $6 \quad$ No. $3 \quad 2012$

The image of complex conjugation in $l$-adic representations associated to automorphic forms

\section{RICHARD TAYLOR}

Betti numbers of graded modules and the multiplicity conjecture in the

non-Cohen-Macaulay case

\section{MATS BOIJ and JONAS SÖDERBERG}

$\mathscr{L}$-invariants and Shimura curves

SAMIT DAsGupta and MATTHEW GREENBERG

On the weak Lefschetz property for powers of linear forms

Juan C. Migliore, Rosa M. Miró-Roig and Uwe Nagel

Resonance equals reducibility for $A$-hypergeometric systems

Mathias Schulze and Uli WaLther

The Chow ring of double EPW sextics

ANDREA FERRETTI

A finiteness property of graded sequences of ideals

Mattias JONSSON and Mircea MustaȚĂ

On unit root formulas for toric exponential sums

Alan Adolphson and SteVen Sperber

Symmetries of the transfer operator for $\Gamma_{0}(N)$ and a character deformation of the 